PROCEEDINGS OF THE

AMERICAN MATHEMATICAL SOCIETY

Volume 134, Number 6, Pages 1743-1751

S 0002-9939(05)08501-1

Article electronically published on December 20, 2005

\title{
SCALAR-VALUED DOMINATED POLYNOMIALS ON BANACH SPACES
}

\author{
GERALDO BOTELHO AND DANIEL M. PELLEGRINO
}

(Communicated by Jonathan M. Borwein)

\begin{abstract}
It is well known that 2-homogeneous polynomials on $\mathcal{L}_{\infty}$-spaces are 2-dominated. Motivated by the fact that related coincidence results are possible only for polynomials defined on symmetrically regular spaces, we investigate the situation in several classes of symmetrically regular spaces. We prove a number of non-coincidence results which makes us suspect that there is no infinite dimensional Banach space $E$ such that every scalar-valued homogeneous polynomial on $E$ is $r$-dominated for every $r \geq 1$.
\end{abstract}

\section{INTRODUCTION}

Starting with the paper by A. Pietsch [23, dominated polynomials and multilinear mappings have been extensively studied in recent years by several authors as an important generalization of absolutely summing operators to the multilinear and polynomial settings. A basic difference between absolutely summing operators and dominated polynomials/multilinear mappings is that, while linear functionals are obviously absolutely summing, multilinear forms and scalar-valued polynomials are not always dominated. For example, regardless of the positive integer $n \geq 2$, from [4, Theorem 4.1 and Example 4.3], we have that the scalar-valued $n$-homogeneous polynomial $\left(a_{j}\right)_{j=1}^{\infty} \in \ell_{2} \longrightarrow \sum_{j=1}^{\infty}\left(a_{j}\right)^{n}$ is not $r$-dominated for any $r \geq 1$. So, it is natural to ask when every $n$-linear form, or every scalar-valued $n$-homogeneous polynomial, on a given Banach space $E$ is $r$-dominated. Only one such coincidence situation was known (Theorem 1.6). In Proposition 2.1 we extend the coincidence and non-coincidence results that hold for $\mathcal{L}_{\infty}$-spaces to the disc algebra $\mathcal{A}$ and to the Hardy space $H^{\infty}$.

After realizing that coincidence theorems are possible only for spaces of polynomials defined on symmetrically regular spaces, in sections 2 and 3 we prove that, even being good candidates a priori, there are non-coincidence theorems on such spaces. It is clear that a non-coincidence theorem for $n$-homogeneous polynomials implies a related non-coincidence theorem for $n$-linear forms and that the converse is not always true. So, for non-coincidence results we concentrated on the polynomial case.

Received by the editors January 18, 2005.

2000 Mathematics Subject Classification. Primary 46G25; Secondary 47B10.

The authors were partially supported by Instituto do Milênio, IMPA. The second author was also supported by CNPq/FAPESQ.

(C)2005 American Mathematical Society Reverts to public domain 28 years from publication 
Our main interest in this paper concerns the existence of an infinite dimensional Banach space $E$ satisfying the following property: every scalar-valued $n$ homogeneous polynomial on $E$ is $r$-dominated for every $r \geq 1$ and every $n \in \mathbb{N}$. The results we prove impel us to conjecture that there is no such space.

\section{Definitions, notation And Basic Results}

Throughout this paper $n$ is a positive integer, $E, F$ and $G$ will stand for (real or complex) Banach spaces and $B_{E}$ denotes the closed unit ball of $E$. The Banach space of all continuous $n$-linear mappings $A: E^{n} \rightarrow F$ will be denoted by $\mathcal{L}\left({ }^{n} E ; F\right)$ and the Banach space of all continuous $n$-homogeneous polynomials $P: E \rightarrow F$ by $\mathcal{P}\left({ }^{n} E ; F\right)$. If $F$ is the scalar field, we write $\mathcal{L}\left({ }^{n} E\right)$ and $\mathcal{P}\left({ }^{n} E\right)$. By $\check{P}$ we mean the unique continuous symmetric $n$-linear mapping associated to the polynomial $P$. For the general theory of multilinear mappings and homogeneous polynomials we refer to S. Dineen [15.

Given $r \in[1, \infty)$, let $\ell_{r}(E)$ be the Banach space of all absolutely $r$-summable sequences $\left(x_{j}\right)_{j=1}^{\infty}$ in $E$ with the norm $\left\|\left(x_{j}\right)_{j=1}^{\infty}\right\|_{r}=\left(\sum_{j=1}^{\infty}\left\|x_{j}\right\|^{r}\right)^{1 / r}$. We denote by $\ell_{r}^{w}(E)$ the Banach space of all weakly $r$-summable sequences $\left(x_{j}\right)_{j=1}^{\infty}$ in $E$ with the norm $\left\|\left(x_{j}\right)_{j=1}^{\infty}\right\|_{w, r}=\sup _{\varphi \in B_{E^{\prime}}}\left\|\left(\varphi\left(x_{j}\right)\right)_{j=1}^{\infty}\right\|_{r} . \quad r=\infty$ is just the case of bounded sequences and in $\ell_{\infty}(E)$ we consider the sup norm. If $0<r<1$ we have $r$-norms instead of norms, and the resulting spaces are complete metrizable topological vector spaces.

1.1. Definition. If $q \leq n r$, an $n$-linear mapping $A \in \mathcal{L}\left({ }^{n} E ; F\right)$ is said to be absolutely $(r ; q)$-summing if $\left(A\left(x_{j}^{1}, \ldots, x_{j}^{n}\right)\right)_{j=1}^{\infty} \in \ell_{r}(F)$ whenever $\left(x_{j}^{k}\right)_{j=1}^{\infty} \in \ell_{q}^{w}(E), k=$ $1, \ldots, n$. The space of all such mappings is denoted by $\mathcal{L}_{a s(r ; q)}\left({ }^{n} E ; F\right)$. See Matos 19 for the characterization by means of inequalities and the resulting norm on $\mathcal{L}_{a s(r ; q)}\left({ }^{n} E ; F\right)$. A polynomial $P \in \mathcal{P}\left({ }^{n} E ; F\right)$ is said to be absolutely $(r ; q)$-summing if $\check{P}$ is absolutely $(r ; q)$-summing. For the space of all such polynomials we write $\mathcal{P}_{a s(r ; q)}\left({ }^{n} E ; F\right)$. A polynomial $P \in \mathcal{P}\left({ }^{n} E ; F\right)$ is absolutely $(r ; q)$-summing if and only if there is $C \geq 0$ such that

$$
\left\|\left(P\left(x_{j}\right)\right)_{j=1}^{k}\right\|_{r} \leq C \cdot\left\|\left(x_{j}\right)_{j=1}^{k}\right\|_{w, q}^{n}
$$

for every finite sequence $x_{1}, \ldots, x_{k} \in E$. The proof of this characterizaton by means of inequalities, as well as the characterization by means of transformations of vector-valued sequences and the resulting norm on $\mathcal{P}_{a s(r ; q)}\left({ }^{n} E ; F\right)$, can be found in Matos [19.

1.2. Polynomials and multilinear mappings of dominated type. An $n$-linear mapping $A \in \mathcal{L}\left({ }^{n} E ; F\right)$ or an $n$-homogeneous polynomial $P \in \mathcal{P}\left({ }^{n} E ; F\right)$ is said to be $r$-dominated if it is absolutely $\left(\frac{r}{n} ; r\right)$-summing. The characterization by means of a Pietsch-type domination, which can be found in Matos [19], justifies the terminology. For the sake of simplicity, we shall denote the space of all $r$-dominated $n$-linear mappings (resp. $n$-homogeneous polynomials) from $E$ into $F$ by $\mathcal{L}_{d, r}\left({ }^{n} E ; F\right)$ (resp. $\left.\mathcal{P}_{d, r}\left({ }^{n} E ; F\right)\right)$.

1.3. The factorization and the linearization methods. Let $\mathcal{I}$ be an operator ideal. According to A. Pietsch 23, a polynomial $P \in \mathcal{P}\left({ }^{n} E ; F\right)$ is said to be of type $\mathcal{P}_{\mathcal{L}(\mathcal{I})}$ (notation: $P \in \mathcal{P}_{\mathcal{L}(\mathcal{I})}\left({ }^{n} E ; F\right)$ ) if there exist a Banach space $G$, a linear operator $u \in \mathcal{I}(E ; G)$ and a polynomial $Q \in \mathcal{P}\left({ }^{n} G ; F\right)$ such that $P=Q \circ u$. 
For $i=1, \ldots, n$, let $\Psi_{i}^{(n)}: \mathcal{L}\left({ }^{n} E ; F\right) \rightarrow \mathcal{L}\left(E ; \mathcal{L}\left({ }^{n-1} E ; F\right)\right)$ represent the canonical isometric isomorphism defined by

$$
\Psi_{i}^{(n)}(A)\left(x_{i}\right)\left(x_{1}, \stackrel{[i]}{\cdot}, x_{n}\right):=A\left(x_{1}, \ldots, x_{n}\right),
$$

where the notation ${ }^{[i]}$. means that the $i$-th coordinate is omitted. A multilinear mapping $A \in \mathcal{L}\left({ }^{n} E ; F\right)$ is said to be of type $[\mathcal{I}]$ if $\Psi_{i}^{(n)}(A) \in \mathcal{I}\left(E ; \mathcal{L}\left({ }^{n-1} E ; F\right)\right)$ for every $i=1, \ldots, n$. A polynomial $P \in \mathcal{P}\left({ }^{n} E ; F\right)$ is of type $[\mathcal{I}]$ (notation: $P \in$ $\left.\mathcal{P}_{[\mathcal{I}]}\left({ }^{n} E ; F\right)\right)$ if $\check{P}$ is of type $[\mathcal{I}]$.

It is well known that $\mathcal{P}_{d, r}\left({ }^{n} E ; F\right)=\mathcal{P}_{\mathcal{L}\left(\Pi_{r}\right)}\left({ }^{n} E ; F\right)$, where $\Pi_{r}$ is the ideal of all absolutely $r$-summing operators.

The following facts are stated for further reference.

1.4. Proposition. Let $\mathcal{I}$ be an operator ideal.

(a) [5] If $\mathcal{P}\left({ }^{n} E ; F\right)=\mathcal{P}_{\mathcal{L}(\mathcal{I})}\left({ }^{n} E ; F\right)$ for some $n$, then $\mathcal{P}\left({ }^{m} E ; F\right)=\mathcal{P}_{\mathcal{L}(\mathcal{I})}\left({ }^{m} E ; F\right)$ for every $m \leq n$.

(b) [17, Corollary 5] If $\mathcal{I}$ is closed and injective, then $\mathcal{P}_{[\mathcal{I}]}\left({ }^{n} E ; F\right)=\mathcal{P}_{\mathcal{L}(\mathcal{I})}\left({ }^{n} E ; F\right)$ for all $n, E$ and $F$.

1.5. Remark. The following simple fact will be used several times: if $F$ is isomorphic to a complemented subspace of $E$ and $\mathcal{P}\left({ }^{n} F\right) \neq \mathcal{P}_{d, r}\left({ }^{n} F\right)$, then $\mathcal{P}\left({ }^{n} E\right) \neq \mathcal{P}_{d, r}\left({ }^{n} E\right)$.

The only coincidence theorem for dominated multilinear mappings and homogeneous polynomials known until now was the following result, which happens to be equivalent to the celebrated Grothendieck's theorem on absolutely summing linear operators from $\ell_{1}$ into $\ell_{2}$.

1.6. Theorem ([3, Theorem 3.3]). If $E$ is an $\mathcal{L}_{\infty}$-space, then $\mathcal{L}\left({ }^{2} E\right)=\mathcal{L}_{d, 2}\left({ }^{2} E\right)$ and, a fortiori, $\mathcal{P}\left({ }^{2} E\right)=\mathcal{P}_{d, 2}\left({ }^{2} E\right)$.

The results proved in 6], 22] yield that Theorem 1.6 is the only coincidence result for $\mathcal{L}_{p}$-spaces, $1 \leq p \leq \infty$.

\subsection{Theorem. (a) ([6, Corollary 4.4(a)]) If $E$ is an $\mathcal{L}_{p}$-space, $1 \leq p<\infty$,} then $\mathcal{P}\left({ }^{n} E\right) \neq \mathcal{P}_{d, r}\left({ }^{n} E\right)$ (hence $\mathcal{L}\left({ }^{n} E\right) \neq \mathcal{L}_{d, r}\left({ }^{n} E\right)$ ) for every $n \geq 2$ and every $r \geq 1$.

(b) ([6, Corollary 4.4] and [22, Corollary 5]) If $E$ is an $\mathcal{L}_{\infty}$-space, then $\mathcal{P}\left({ }^{n} E\right) \neq$ $\mathcal{P}_{d, r}\left({ }^{n} E\right)$ (hence $\mathcal{L}\left({ }^{n} E\right) \neq \mathcal{L}_{d, r}\left({ }^{n} E\right)$ ) for every $n \geq 3$ and every $r \geq 1$ and $\mathcal{P}\left({ }^{2} E\right) \neq \mathcal{P}_{d, r}\left({ }^{2} E\right)$ (hence $\mathcal{L}\left({ }^{2} E\right) \neq \mathcal{L}_{d, r}\left({ }^{2} E\right)$ ) for every $r<2$.

\section{Symmetrically REgular SPACES}

A bounded linear operator $u: E \rightarrow E^{\prime}$ is symmetric if $u(x)(y)=u(y)(x)$ for every $x, y \in E$. A Banach space $E$ is said to be symmetrically regular if every symmetric bounded linear operator from $E$ into $E^{\prime}$ is weakly compact. Assume that $\mathcal{P}\left({ }^{n} E\right)=\mathcal{P}_{d, r}\left({ }^{n} E\right)$ for some $n$ and some $r$. From Proposition 1.4(a) we have that $\mathcal{P}\left({ }^{2} E\right)=\mathcal{P}_{d, r}\left({ }^{2} E\right)$ and from [3, Lemma 3.4] we have that every symmetric bounded linear operator from $E$ into $E^{\prime}$ is $r$-summing. Since $r$-summing operators are weakly compact it follows that $E$ is symmetrically regular. So, coincidence theorems should be sought on symmetrically regular spaces.

Some of the most common non-reflexive symmetrically regular spaces are: $C^{*}$ algebras, the disc algebra $\mathcal{A}$, the Hardy space $H^{\infty}$, the James space $J$ and its dual $J^{\prime}$, and Banach spaces of type 2 . 
Since commutative $C^{*}$-algebras with unit are $C(K)$-spaces, this case was completely solved by Theorems 1.6 and $1.7(\mathrm{~b})$. If $E$ is either a non-commutative $C^{*}$ algebra with unit, or $J$ or $J^{\prime}$, it is easy to see that $E$ contains a complemented copy of a Hilbert space (a proof that $J$ contains a complemented copy of $\ell_{2}$ can be found in [16. Corollary 2.d.4]). Since Hilbert spaces are $\mathcal{L}_{2}$-spaces, by Remark 1.5 and Theorem 1.7(a) it follows that $\mathcal{P}\left({ }^{n} E\right) \neq \mathcal{P}_{d, r}\left({ }^{n} E\right)$ for every $n \geq 2$ and every $r \geq 1$.

The cases of $\mathcal{A}$ and $H^{\infty}$ are interesting for two reasons: (i) they provide two new coincidence theorems, (ii) like several other aspects of Banach space theory, the next proposition shows that $\mathcal{A}$ and $H^{\infty}$ behave exactly like $\mathcal{L}_{\infty}$-spaces with respect to spaces of dominated multilinear forms and scalar-valued homogeneous polynomials, although they are not $\mathcal{L}_{\infty}$-spaces (see [9, p. 4] and [13, Corollary 23.9.2]). If $\pi$ denotes the unit circle, $\mathcal{A}$ can be regarded as a subspace of $C(\pi)$ and $H^{\infty}$ can be regarded as a subspace of $L_{\infty}(\pi)$ (see [20]).

2.1. Proposition. Let $n \in \mathbb{N}$ and $r \geq 1$. The following assertions are equivalent:

(a) $\mathcal{P}\left({ }^{n} \mathcal{A}\right)=\mathcal{P}_{d, r}\left({ }^{n} \mathcal{A}\right)$

(b) $\mathcal{P}\left({ }^{n} H^{\infty}\right)=\mathcal{P}_{d, r}\left({ }^{n} H^{\infty}\right)$

(c) $\mathcal{P}\left({ }^{n} E\right)=\mathcal{P}_{d, r}\left({ }^{n} E\right)$ for some $\mathcal{L}_{\infty}$-space $E$.

(d) $\mathcal{P}\left({ }^{n} E\right)=\mathcal{P}_{d, r}\left({ }^{n} E\right)$ for every $\mathcal{L}_{\infty}$-space $E$.

(e) $n=2$ and $r \geq 2$.

In particular, $\mathcal{P}\left({ }^{2} \mathcal{A}\right)=\mathcal{P}_{d, 2}\left({ }^{2} \mathcal{A}\right)$ and $\mathcal{P}\left({ }^{2} H^{\infty}\right)=\mathcal{P}_{d, 2}\left({ }^{2} H^{\infty}\right)$. Moreover, the assertions above remain equivalent if the spaces of $n$-homogeneous polynomials are replaced by spaces of $n$-linear forms. In particular, $\mathcal{L}\left({ }^{2} \mathcal{A}\right)=\mathcal{L}_{d, 2}\left({ }^{2} \mathcal{A}\right)$ and $\mathcal{L}\left({ }^{2} H^{\infty}\right)=\mathcal{L}_{d, 2}\left({ }^{2} H^{\infty}\right)$.

Proof. (c) $\Leftrightarrow$ (d) follows from a standard localization argument, (e) $\Rightarrow$ (d) is Theorem 1.6 and $(\mathrm{d}) \Rightarrow(\mathrm{e})$ is Theorem $1.7(\mathrm{~b})$.

(a) $\Rightarrow$ (c) Since $\mathcal{A}$ contains isomorphic copies of $c_{0}$ (see [20) and is separable (because it has a basis 20, Proposition 10.4]), it follows from [18, Theorem 2.f.5] that $\mathcal{A}$ contains complemented copies isomorphic to $c_{0}$. The result follows from Remark 1.5.

(b) $\Rightarrow$ (c) $H^{\infty}$ is a dual space (see [20, p.11]) which contains an isomorphic copy of $c_{0}$ (in fact, $H^{\infty}$ contains an isomorphic copy of $\mathcal{A}[20$, p. 4]), so by one of the results due to C. Bessaga-A. Pełczyński (see [18, Proposition 2.e.8]) we have that $H^{\infty}$ contains a complemented isomorphic copy of $\ell_{\infty}$. Again, the result follows from Remark 1.5.

(e) $\Rightarrow$ (a) Since $\mathcal{P}_{d, r}\left({ }^{n} E ; F\right) \subseteq \mathcal{P}_{d, s}\left({ }^{n} E ; F\right)$ whenever $r \leq s$, it suffices to show that $\mathcal{P}\left({ }^{2} \mathcal{A}\right)=\mathcal{P}_{d, 2}\left({ }^{2} \mathcal{A}\right)$. Given $P \in \mathcal{P}\left({ }^{2} \mathcal{A}\right)$, by a result due to J. Bourgain 8 , p. 946], $\check{P}$ can be extended to a bilinear form $B \in \mathcal{L}\left({ }^{2} C(\pi)\right)$, which is 2-dominated by Theorem 1.6. The result follows because restrictions of 2-dominated mappings are 2-dominated and because $P$ is $r$-dominated if and only if $\check{P}$ is.

(e) $\Rightarrow$ (b) Repeat the proof of (e) $\Rightarrow$ (a) using another result due to J. Bourgain [10. Corollary 3.10] which asserts that every continuous bilinear form on $H^{\infty}$ can be extended to a continuous bilinear form on $L_{\infty}(\pi)$.

The proof can be obviously adapted to multilinear mappings.

Let us introduce some terminology in order to investigate non-coincidence situations in Banach spaces of non-trivial type. According to J. Castillo-F. Sánchez [12], we say that a Banach space $E \in C_{r}$ if every sequence $\left(x_{j}\right)_{j=1}^{\infty} \in \ell_{r}^{w}(E)$ is norm null. 
2.2. Proposition. Let $E$ be a Banach space of non-trivial type such that $E \notin C_{r}$ for some $r>1$. Then there is $N \in \mathbb{N}$ such that $\mathcal{P}\left({ }^{n} E\right) \neq \mathcal{P}_{\text {as }(s ; r)}\left({ }^{n} E\right)$ for every $s$ and every $n \geq N$. In particular, $\mathcal{P}\left({ }^{n} E\right) \neq \mathcal{P}_{d, r}\left({ }^{n} E\right)$ for every $n \geq N$.

Proof. By [14, Corollary 13.7] we know that $E^{\prime}$ has some non-trivial type $p>1$. Since $E \notin C_{r}$, there exists a weakly $r$-summable sequence in $E$ which is not norm null. Hence, passing to a subsequence if necessary, there exists a seminormalized sequence $\left(z_{j}\right) \in \ell_{r}^{w}(E)$. It is obvious that $\left(z_{j}\right)$ is weakly convergent to zero. Given $n>\frac{p}{p-1}$, choose $\left(a_{j}\right) \in \ell_{\infty},\left(a_{j}\right) \notin \ell_{s}$, and use M. Valdivia 24] to produce a polynomial $P \in \mathcal{P}\left({ }^{n} E\right)$ such that $P\left(x_{j}\right)=a_{j}$ for every $j$, where $\left(x_{j}\right)$ is some subsequence of $\left(z_{j}\right)$. In this fashion $\left(x_{j}\right) \in \ell_{r}^{w}(E)$ but $\left(P\left(x_{j}\right)\right)=\left(a_{j}\right) \notin \ell_{s}$. This proves that $P$ is not absolutely $(s ; r)$-summing.

2.3. Remark. The requirements of Proposition 2.2 are satisfied, for example: (i) by any Banach space of non-trivial type containing an isomorphic copy of some $\ell_{p}$, $1<p<\infty$ (using the canonical unit vectors it is easy to see that $\ell_{p} \notin C_{r}$ whenever $r \geq p^{\prime}$, where $\frac{1}{p}+\frac{1}{p^{\prime}}=1$ ); (ii) by any infinite-dimensional superreflexive Banach space (it is well known that $E$ superreflexive $\Rightarrow E$ uniformly convexifiable $\Rightarrow E$ has non-trivial type, and by [12, p. 118] it follows that $E \notin C_{r}$ for some $r>1$ ), in particular by the 2-convexified Tsirelson space $T_{2}$ (see [11) which happens to be a type 2 space not containing any copy of $\ell_{p}, 1<p<\infty$.

Another class of symmetrically regular spaces deserves a specific section.

\section{Q-REFLEXIVE SPACES}

By $\mathcal{P}_{w}\left({ }^{n} E\right)$ we denote the set of all continuous scalar-valued $n$-homogeneous polynomials on $E$ which are weakly continuous on bounded sets.

3.1. Lemma. If $\mathcal{P}\left({ }^{n} E\right)=\mathcal{P}_{w}\left({ }^{n} E\right)$ for some $n \geq 2$, then $E$ is symmetrically regular.

Proof. Let $\mathcal{K}$ and $\mathcal{W}$ denote the ideals of compact and weakly compact operators, respectively. By 2, Theorem 2.9] it follows that $\mathcal{P}\left({ }^{n} E\right)=\mathcal{P}_{[\mathcal{K}]}\left({ }^{n} E\right)$, and consequently we have that $\mathcal{P}\left({ }^{n} E\right)=\mathcal{P}_{\mathcal{L}(\mathcal{K})}\left({ }^{n} E\right)$ by Proposition 1.4(b). Using Proposition 1.4(a) and $(\mathrm{b})$ once more we get

$$
\mathcal{P}\left({ }^{2} E\right)=\mathcal{P}_{\mathcal{L}(\mathcal{K})}\left({ }^{2} E\right) \subseteq \mathcal{P}_{\mathcal{L}(\mathcal{W})}\left({ }^{2} E\right)=\mathcal{P}_{[\mathcal{W}]}\left({ }^{2} E\right),
$$

showing that $\mathcal{P}\left({ }^{2} E\right)=\mathcal{P}_{[\mathcal{W}]}\left({ }^{2} E\right)$, that is, $E$ is symmetrically regular.

Given a Banach space $E$ and $n \in \mathbb{N}$, by $A B_{n}: \mathcal{P}\left({ }^{n} E\right) \rightarrow \mathcal{P}\left({ }^{n} E^{\prime \prime}\right)$ we denote the Aron-Berner extension operator (cf. [15, Proposition 1.53]) and by $\widehat{\otimes}_{n, \pi, s} E$ we mean the $n$-fold symmetric projective tensor product of $E$. Define

$$
J_{n}: \widehat{\otimes}_{n, \pi, s} E^{\prime \prime} \longrightarrow \mathcal{P}\left({ }^{n} E\right)^{\prime} ;\left[J_{n}\left(\otimes_{n} x\right)\right](P)=\left[A B_{n}(P)\right](x)
$$

for all $x \in E^{\prime \prime}$ and $P \in \mathcal{P}\left({ }^{n} E\right)$. According to [15, Definition 2.44], we say that $E$ is $Q$-reflexive if $J_{n}$ is an isomorphism for all $n$. The following features of Q-reflexive spaces justify our interest: if $E$ is a Q-reflexive space and $E^{\prime \prime}$ has the approximation property, then $E$ is symmetrically regular (combine M. Venkova 25, Proposition 2.2] and Lemma 3.1), $E$ has no non-trivial type [25, Proposition 4.7] and $E$ is not an $\mathcal{L}_{p}$-space, $1 \leq p \leq \infty$ ( $E$ is not an $\mathcal{L}_{p}$-space, $1<p<\infty$, because such spaces have some non-trivial type and $E$ is neither an $\mathcal{L}_{1}$ nor an $\mathcal{L}_{\infty}$-space because such spaces have the Dunford-Pettis property [7, Corollary 1.30] whereas $E$ fails this property [25, Proposition 3.6]). 
Let us deal with the reflexive case first. It is well known that a reflexive space $E$ is Q-reflexive if and only if $\mathcal{P}\left({ }^{n} E\right)$ is reflexive for all $n \in \mathbb{N}$ [1, p. 1011]. So, the prototype of a reflexive Q-reflexive space is Tsirelson's original space $T^{*} . T^{*}$ and other Tsirelson-like spaces, including the already mentioned space $T_{2}$, are treated in the next result.

3.2. Theorem. If $E$ has an unconditional basis, then $\mathcal{P}\left({ }^{n} E\right) \neq \mathcal{P}_{d, 1}\left({ }^{n} E\right)$ for every $n \geq 2$.

Proof. It suffices to show that $\mathcal{P}\left({ }^{2} E\right) \neq \mathcal{P}_{d, 1}\left({ }^{2} E\right)$. Let $\left(x_{j}\right)$ be a normalized unconditional basis of $E$. Then there exists $\rho>0$ such that if $x=\sum_{j=1}^{\infty} a_{j} x_{j}$, then

$$
\left\|\sum_{j=1}^{k} \varepsilon_{j} a_{j} x_{j}\right\| \leq \rho\|x\|, \text { for every } k \text { and every } \varepsilon_{j}= \pm 1 .
$$

Assume that $\mathcal{P}\left({ }^{2} E\right)=\mathcal{P}_{d, 1}\left({ }^{2} E\right)$. It is plain that there exists $C$ such that $\|P\|_{d, 1} \leq$ $C\|P\|$ for all $P \in \mathcal{P}\left({ }^{2} E\right)$. Given $k \in \mathbb{N}$ and a finite sequence of scalars $\mu_{1}, \ldots, \mu_{k}$ so that $\sum_{j=1}^{k}\left|\mu_{j}\right|^{2}=1$, consider the scalar-valued 2-homogeneous polynomial $Q$ on $E$ defined by

$$
x=\sum_{j=1}^{\infty} a_{j} x_{j} \in E \longrightarrow Q(x)=\sum_{j=1}^{k}\left|\mu_{j}\right|^{2} a_{j}^{2} .
$$

For all $x \in E$ it follows immediately that $|Q(x)| \leq \sum_{j=1}^{k}\left|\mu_{j}\right|^{2}\left|a_{j}\right|^{2} \leq \rho^{2}\|x\|^{2}$. Proceeding as in the proof of [21, Theorem 5] we obtain

$$
\sum_{j=1}^{k}\left|a_{j}\right|^{2} \leq C \rho^{4}\|x\|^{2}, \text { for every } k \in \mathbb{N} .
$$

Fix $k \in \mathbb{N}$ and define

$$
P: E \longrightarrow \mathbb{K} ; P(x)=\sum_{j=1}^{k} a_{j}^{2}, \text { where } x=\sum_{j=1}^{\infty} a_{j} x_{j} .
$$

Thus $P$ is 1-dominated. Given $x=\sum_{j=1}^{\infty} a_{j} x_{j} \in E,|P(x)| \leq \sum_{j=1}^{k}\left|a_{j}\right|^{2} \leq$ $C \rho^{4}\|x\|^{2}$, which shows that $\|P\| \leq C \rho^{4}$, therefore $\|P\|_{d, 1} \leq C^{2} \rho^{4}$. Then

$$
\begin{gathered}
\sum_{j=1}^{k}\left|a_{j}\right|=\sum_{j=1}^{k}\left|a_{j}^{2}\right|^{\frac{1}{2}}=\sum_{j=1}^{k}\left|P\left(a_{j} x_{j}\right)\right|^{\frac{1}{2}} \leq\|P\|_{d, 1}^{\frac{1}{2}} \cdot\left\|\left(a_{j} x_{j}\right)_{j=1}^{k}\right\|_{w, 1} \\
\leq C \rho^{2} 2 \max _{\varepsilon_{j}= \pm 1}\left\|\sum_{j=1}^{k} \varepsilon_{j} a_{j} x_{j}\right\| \leq 2 C \rho^{3}\|x\| .
\end{gathered}
$$

Since $k$ is arbitrary, we have that for every $x \in E$,

$$
\|x\|=\left\|\sum_{j=1}^{\infty} a_{j} x_{j}\right\| \leq \sum_{j=1}^{\infty}\left|a_{j}\right| \leq 2 C \rho^{3}\|x\|,
$$

which shows that $E$ is isomorphic to $\ell_{1}$. This contradiction - by Remark 1.5 and Theorem 1.7(a), $E$ cannot be isomorphic to $\ell_{1}$ - completes the proof. 
Turning to non-reflexive Q-reflexive spaces, we focus our attention on the James space $T_{J}^{*}$ modelled on $T^{*}$ (see R. Aron-S. Dineen [1]). $T_{J}^{*}$ must be studied separately because it is a Q-reflexive space [1, Proposition 15] which has no unconditional basis (because $T_{J}^{*}$ is non-reflexive and $\left(T_{J}^{*}\right)^{\prime \prime}$ is separable [18, Theorem 1.c.12]), and its second dual has the approximation property $\left(T_{J}^{*}\right.$ and all its duals have basis 1 , p. 1021]).

Let $\left(e_{j}\right)_{j=1}^{\infty}$ denote the canonical unit vectors of sequence spaces. By 1 , Proposition 8] we know that $\left(e_{j}\right)_{j=1}^{\infty}$ is a monotone basis for $T_{J}^{*}$. Then, for $x=\sum_{j=1}^{\infty} a_{j} e_{j} \in$ $T_{J}^{*}$ we have that $\|x\| \geq\left\|\sum_{j=1}^{k} a_{j} e_{j}\right\|$ for every $k \in \mathbb{N}$.

3.3. Theorem. $\mathcal{P}\left({ }^{n} T_{J}^{*}\right) \neq \mathcal{P}_{\text {as }(q ; 1)}\left({ }^{n} T_{J}^{*}\right)$ for every $n \geq 2$ and every $q<1$. In particular, $\mathcal{P}\left({ }^{n} T_{J}^{*}\right) \neq \mathcal{P}_{d, r}\left({ }^{n} T_{J}^{*}\right)$ whenever $n \geq 2$ and $r<n$.

Proof. Assume that there is $q<1$ such that $\mathcal{P}\left({ }^{n} T_{J}^{*}\right)=\mathcal{P}_{\text {as }(q ; 1)}\left({ }^{n} T_{J}^{*}\right)$. Then there exists $C$ such that $\|P\|_{a s(q ; 1)} \leq C\|P\|$ for all $P \in \mathcal{P}\left({ }^{n} T_{J}^{*}\right)$. Fix $k \in \mathbb{N}$ and choose scalars $\mu_{k}, \ldots, \mu_{2 k}$ such that $\sum_{j=k}^{2 k}\left|\mu_{j}\right|^{s}=1$, where $s=\frac{1}{q}$. Consider the $n$-homogeneous polynomial on $T_{J}^{*}$ defined by

$$
P(x)=\sum_{j=k}^{2 k}\left|\mu_{j}\right|^{\frac{1}{q}} a_{j}^{n}, \text { where } x=\sum_{j=1}^{\infty} a_{j} e_{j} .
$$

Using the monotonicity of the basis $\left(e_{j}\right)_{j=1}^{\infty}$ we have that $|P(x)| \leq 2^{n}\|x\|^{n}$ for every $x \in T_{J}^{*}$. Hence $\|P\|_{a s(q ; 1)} \leq 2^{n} C$. By [1, Corollary 10] we have that

$$
\begin{gathered}
\left(\sum_{j=k}^{2 k}\left|\mu_{j}\right|\left|a_{j}\right|^{n q}\right)^{\frac{1}{q}}=\left(\left.\left.\sum_{j=k}^{2 k}\left|a_{j}^{n}\right| \mu_{j}\right|^{\frac{1}{q}}\right|^{q}\right)^{\frac{1}{q}}=\left(\sum_{j=k}^{2 k}\left|P\left(a_{j} e_{j}\right)\right|^{q}\right)^{\frac{1}{q}} \\
\leq\|P\|_{a s(q ; 1)} 2^{n} \max _{\varepsilon_{j}= \pm 1}\left\|\sum_{j=k}^{2 k} \varepsilon_{j} a_{j} e_{j}\right\|^{n} \\
\leq 4^{n} C\left[\max _{\varepsilon_{j}= \pm 1}\left(\sup _{k \leq j \leq 2 k}\left|\varepsilon_{j} a_{j}\right|\right)\right]^{n}=4^{n} C\left(\sup _{k \leq j \leq 2 k}\left|a_{j}\right|\right)^{n} .
\end{gathered}
$$

Combining this estimate with

$$
\begin{aligned}
& \left(\sum_{j=k}^{2 k}\left|a_{j}\right|^{\frac{s}{s-1} n q}\right)^{\frac{s-1}{s}}=\left\|\left(\left|a_{j}\right|^{n q}\right)_{j=k}^{2 k}\right\|_{\frac{s}{s-1}} \\
& \leq \sup \left\{\sum_{j=k}^{2 k}\left|\nu_{j}\right|\left|a_{j}\right|^{n q} ; \sum_{j=k}^{2 k}\left|\nu_{j}\right|^{s}=1\right\},
\end{aligned}
$$

we get that

$$
\left(\sum_{j=k}^{2 k}\left|a_{j}\right|^{\frac{s}{s-1} n q}\right)^{\frac{s-1}{s}} \leq\left[4^{n} C\left(\sup _{k \leq j \leq 2 k}\left|a_{j}\right|\right)^{n}\right]^{q} .
$$

Putting $r=\frac{s}{s-1} n q$ we have just proved that

$$
\left(\sum_{j=k}^{2 k}\left|a_{j}\right|^{r}\right)^{\frac{1}{r}} \leq 4 C^{\frac{1}{n}} \sup _{k \leq j \leq 2 k}\left|a_{j}\right|, \text { for every } k \in \mathbb{N} .
$$

From the proof of [1, Corollary 11] we know that the above estimate is not possible, so the proof is complete. 
The techniques used in the proofs of Theorem 3.3 and [21, Theorem 5] can be combined to prove the following results (the symbol $\cot F$ stands for the cotype constant of the Banach space $F$ ).

3.4. Theorem. Let $F$ be an infinite dimensional Banach space and $n \geq 2$.

(a) If $F$ finitely factors the formal inclusion $\ell_{p} \hookrightarrow \ell_{\infty}$ for some (or, equivalently, for all) $0<\delta<1$, then $\mathcal{P}\left({ }^{n} T_{J}^{*} ; F\right) \neq \mathcal{P}_{a s(q ; 1)}\left({ }^{n} T_{J}^{*} ; F\right)$ for every $q<p$. In particular, $\mathcal{P}\left({ }^{n} T_{J}^{*} ; F\right) \neq \mathcal{P}_{\text {as }(q ; 1)}\left({ }^{n} T_{J}^{*} ; F\right)$ for every $q<2$.

(b) If $F$ is of finite cotype, then $\mathcal{P}\left({ }^{n} T_{J}^{*} ; F\right) \neq \mathcal{P}_{d, r}\left({ }^{n} T_{J}^{*} ; F\right)$ for every $r<n$. $\cot F$. In particular, $\mathcal{P}\left({ }^{n} T_{J}^{*} ; F\right) \neq \mathcal{P}_{d, r}\left({ }^{n} T_{J}^{*} ; F\right)$ for every $r<2 n$ and every $F$ of finite cotype.

\section{REFERENCES}

[1] R. M. Aron and S. Dineen. Q-reflexive Banach spaces, Rocky Mountain J. Math. 27 (1997), 1009-1025. MR:1627646 (99h:46010)

[2] R. M. Aron, C. Hervés and M. Valdivia. Weakly continuous mappings on Banach spaces, J. Functional Analysis 52 (1983), 189-204. MR0707203 (84g:46066)

[3] G. Botelho. Cotype and absolutely summing multilinear mappings and homogeneous polynomials, Proc. Roy. Irish Acad. Sect. A 97 (1997), 145-153. MR1645283 (99i:46006)

[4] G. Botelho. Almost summing polynomials, Math. Nachr. 211 (2000), 25-36. MR.1743489 (2001d:46069)

[5] G. Botelho. Ideals of polynomials generated by weakly compact operators, Note Mat. 25 (2005).

[6] G. Botelho and D. Pellegrino. Dominated polynomials on $\mathcal{L}_{p}$-spaces, Arch. Math. 83 (2004), 364-370. MR2096810 (2005e:46076)

[7] J. Bourgain. New classes of $\mathcal{L}_{p}$-spaces, Lecture Notes in Math. 889, 1981. MR0639014 (83j:46028)

[8] J. Bourgain. New Banach space properties of certain spaces of analytic functions, Proc. Internat. Congress of Mathematicians, vol. II. Warzawa, 1983, 945-951. MR0804748 (86m:46050)

[9] J. Bourgain. New Banach space properties of the disc algebra and $H^{\infty}$, Acta. Math. 152 (1984), 1-48. MR0736210 (85j:46091)

[10] J. Bourgain. Bilinear forms on $H^{\infty}$ and bounded bianalytic functions, Trans. Amer. Math. Soc. 286 (1984), 313-337. MR0756042 (86c:46060)

[11] P. G. Casazza and T. J. Shura. Tsirelson's spaces, Lecture Notes in Math. 1363 (1989). MR0981801 (90b:46030)

[12] J. Castillo and F. Sánchez. Remarks on some basic properties of Tsirelson's space, Note Mat. XIII (1993), 117-122. MR1283523 (95f:46011)

[13] A. Defant and K. Floret. Tensor Norms and Operator Ideals, North-Holland Mathematics Studies 176, North-Holland, 1993. MR.1209438(94e:46130)

[14] J. Diestel, H. Jarchow and A. Tonge. Absolutely summing operators, Cambridge University Press, Cambridge, 1995. MR1342297 (96i:46001)

[15] S. Dineen. Complex Analysis on Infinite Dimensional Spaces, Springer-Verlag, London, 1999. MR1705327 (2001a:46043)

[16] H. Fetter and B. Gamboa. The James forest, London Mathematical Society Lecture Notes Series, 236, 1997. MR1474498 (98k:46013)

[17] M. González and J. Gutiérrez. Injective factorization of holomorphic mappings, Proc. Amer. Math. Soc. 127 (1999), 1715-1721. See also Erratum in vol. 129 (2001), 1255-1256. MR.1610897 (99i:46033) MR.1814156 (2002a:46059)

[18] J. Lindenstrauss and L. Tzafriri. Classical Banach Spaces I and II, Springer-Verlag, 1996.

[19] M. Matos. Absolutely summing holomorphic mappings, An. Acad. Brasil. Ciênc. 68 (1996), 1-13. MR 1752625(2001c:46086)

[20] A. Pełczyński. Banach spaces of analytic functions and absolutely summing operators, CBMS Regional Conf. Series 30, Amer. Math. Soc., 1977. MR0511811 (58:23526)

[21] D. Pellegrino. Cotype and absolutely summing homogeneous polynomials in $\mathcal{L}_{p}$ spaces, Studia Math. 157 (2003), 121-131. MR.1980709 (2004f:46019) 
[22] D. Pellegrino. On scalar-valued nonlinear absolutely summing mappings, Ann. Polon. Math. 83 (2004), 281-288. MR.2111715 (2005h:46022)

[23] A. Pietsch. Ideals of multilinear functionals, Proceedings of the Second International Conference on Operator Algebras, Ideals and Their Applications in Theoretical Physics, 185-199, Teubner-Texte, Leipzig, 1983. MR0763541

[24] M. Valdivia. Complemented subspaces and interpolation properties in spaces of polynomials, J. Math. Anal. Appl. 208 (1997), 1-30. MR1440340 (99d:46026)

[25] M. Venkova. Properties of Q-reflexive Banach spaces, J. Math. Anal. Appl. 264 (2001), 96106. MR.1868330(2003a:46034)

Faculdade de Matemática, Universidade Federal de Uberlândia, 38.400-902, UberLÂNDIA, BRAZIL

E-mail address: botelho@ufu.br

Departamento de Matemática e Estatística, Universidade Federal de Campina Grande, 58.109-970, Campina Grande Brazil

E-mail address: pellegrino@dme.ufcg.edu.br 